\section{$\underset{\substack{\text { hommes } \\ \text { \& migrations }}}{ }$}

\section{Hommes \& migrations}

Revue française de référence sur les dynamiques

migratoires

\section{$1305 \mid 2014$}

L'exil chilien en France

\title{
12 years a slave
}

Film britannique de Steeve McQueen

\section{Anaïs Vincent}

\section{Q OpenEdition \\ 1 Journals}

\section{Édition électronique}

URL : http://journals.openedition.org/hommesmigrations/2770

DOI : 10.4000/hommesmigrations. 2770

ISSN : 2262-3353

\section{Éditeur}

Musée national de l'histoire de l'immigration

\section{Édition imprimée}

Date de publication : 1 janvier 2014

Pagination : 193-194

ISBN : 978-2919-040261

ISSN : 1142-852X

\section{Référence électronique}

Anaiis Vincent, « 12 years a slave », Hommes \& migrations [En ligne], 1305 | 2014, mis en ligne le 16 juillet 2014, consulté le 22 septembre 2020. URL : http://journals.openedition.org/hommesmigrations/ 2770 ; DOI : https://doi.org/10.4000/hommesmigrations.2770 


\section{CINÉMA}

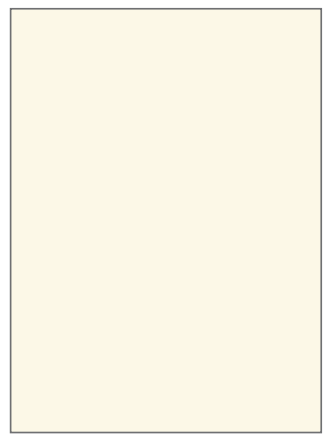

\section{Steeve McQueen}

12 Years a Slave

Film britannique

La sortie en salle du dernier film du réalisateur britannique Steeve Mc Queen, Twelve Years a Slave n'est pas passée inaperçue.

Multinominé aux Oscars, ce troisième long-métrage est sorti grand vainqueur de la compétition, récompensé pour le meilleur film de l'année, la meilleure adaptation et le meilleur rôle féminin.

Le scénario a été adapté du récit de Solomon Northrup, l'un des rares esclaves a avoir retrouvé la liberté. Solomon est un jeune père de famille noir citoyen de l'État de New York, violoniste libre. Drogué à son insu, il est kidnappé en 1841 à Washington pour être vendu à un marchand d'esclaves et libéré en 1853. Il est acheté tour à tour par différents négriers et raconte avec violence ses douze années de captivité avec une précision glaçante. Avec lui nous sommes plongés dans les méandres de l'obscurantisme américain de la fin du XIXe siècle. Devoir mémoriel, les films sur cette période sombre de I'histoire se succèdent à Hollywood. Après le sanglant et caricatural Django Unchained de Tarantino, Steeve McQueen aborde la question de l'esclavage avec une grande virtuosité. En quoi sa vision diffère-t-elle de ses prédécesseurs ?

La première scène du film s'ouvre sur un plan des esclaves figés comme posant dans un champ de cannes à sucre. Reconstitution d'une photographie documentant un passé sordide.
La première pierre est posée. Le ton est donné.Avecexactitude,SteeveMcQueen colle au récit de Solomon Northrup. Les Slaves narratives, ces récits d'esclaves furent très populaires pour soutenir les thèses abolitionnistes puis tombèrent progressivement dans l'oubli jusqu'à ce qu'ils soient réétudiés par les historiens à partir des années 1960, constituant des témoignages précieux d'une période si trouble et si difficile à expier. En 1841, John Tyler est élu dixième président des États-Unis. Depuis 1787 l'ordonnance du Nord-Ouest interdit l'esclavage dans le territoire du NordOuest. L'abolitionnisme porté par une vague internationale touche les États-Unis. La traite négrière est abolie officiellement en 1808, même si les contrebandiers la poursuivent clandestinement pendant plusieurs années. À l'issu de la Guerre de sécession l'abolition de l'esclavage est proclamée le 18 décembre 1865 .

Mais en 1841 , les grandes exploitations agricoles du sud continuent à exploiter en toute impunité une main-d'œuvre d'esclaves noirs. Pourtant Steeve McQueen n'oppose jamais Sud et Nord. Il sait que la réalité était plus complexe. La condition des Noirs était loin d'être idyllique dans les États du Nord et parfois la situation de certains au Sud étaient plus vivable.

Dès son enlèvement, les ravisseurs falsifient l'identité de Solomon. II devient Pratt et comprend très vite que pour survivre, il est vital d'oublier son passé Broyé par ce commerce, dans un atroce processus de réification, il raconte cette soumission. Son souffle, cette respiration vitale ponctue le récit et laisse 
retentir le silence de l'innommable. II nous prend à témoin dans un regard caméra inquisiteur.

Le cinéaste retranscrit avec précision la violence physique, le sifflement du fouet sur les corps engourdis par le labeur, la chair à vif, décrits dans ses mémoires. II revient avec force détails sur l'épisode si angoissant de la pesée et la façon dont les hommes étaient roués de coups s'ils n'avaient pas atteint leurs quotas de récolte.

Nul manichéisme pourtant, l'ambivalence règne. Son violon qui le rattache à sa vie d'homme libre et lui permet de gagner quelques pièces en jouant occasionnellement pour les réceptions du juge, devient instrument de torture lorsque Edwins Epps (Michael Fassbender), le propriétaire de l'exploitation de coton de Bayou Boeuf, lui demande de jouer pour faire danser ses camarades épuisés dans un bal grotesque et humiliant. Dans un excès de désespoir, il le brise.

Le réalisateur choisit un des rares récits décrivant la condition des femmes avec très peu de pudeur. La jeune Patsey est doublement esclave. Le soir, la récolte finie, elle est violée par l'ignoble Edwins Epps. Violences physiques et psychologiques se mêlent.

Mais, comme l'affirme Henri Louis Gate, spécialiste de la black culture, et conseillé sur ce film, Steeve McQueen n'est jamais caricatural. Il déshumanise à la fois les esclaves et les propriétaires. Comme dans ses précédents films, il dépeint avec noirceur les sentiments les plus vils de l'humanité. Encore dans certaines salles depuis sa sortie le 22 janvier dernier, 12 Years a Slave sera disponible en DVD le 28 mai prochain. 


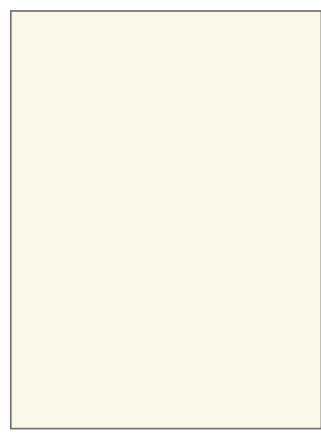

\section{Dyana Gaye}

\section{Des étoiles}

Film franco-sénégalais

Le premier long-métrage de la réalisatrice Dyana Gaye, Des étoiles, est sorti le 29 janvier dernier en salles. Sélectionné aux festivals Premier Plans d'Angers et de Toronto, ce film ambitieux raconte les parcours de jeunes migrants entre Dakar, New York et Turin. Cette narration triangulaire nous fait partager l'expérience de l'exil de ses trois protagonistes.

Sophie part de Dakar pour rejoindre son mari Abdoulaye à Turin. Mais ce dernier est parti tenter sa chance à New York. Pour les obsèques de son père, Thierno, un jeune New-Yorkais, découvre pour la première fois avec sa mère d'origine sénégalaise Dakar. On passe d'une ville à l'autre, d'une histoire à l'autre, d'une langue à l'autre, dans un mouvement fluide sans heurts ni effets. Le choix de ces trois villes est apparu tout naturellement à la réalisatrice dont la mère est métisse d'origine franco-italomalienne-sénégalaise et le père sénégalais immigré en France. Elle a souhaité construire une circulation entre trois villes familières. Turin, cité industrielle a eu massivement recourt à l'immigration. Cependant, depuis quelques années, le flux s'inverse. En cause le durcissement des lois et la crise économique. Les immigrés touchés par le chômage sont expulsés. La Ligue du Nord, parti fasciste, gagne du terrain. Loin de cette vision dramatique, la réalisatrice choisit de mettre en scène un parcours d'intégration sans fausse note, à l'image de celui du jeune Rachid Khadiri Abdelmoura, un étudiant marocain vendeur de briquets, tout juste devenu docteur en ingénierie de la prestigieuse université polytechnique de Turin. Rachid n'a pas eu pourtant la chance de Sophie qui, dès son arrivée, est prise en charge par une association d'aide aux sans-papiers et est épaulée par la solidarité communautaire. En Italie, le réseau associatif sénégalais est très actif et facilite l'intégration des nouveaux arrivants dans la société. Très vite, elle trouve un logement, apprend la langue et un travail et tombe amoureuse. Vision positive et un peu idéaliste d'une immigration réussie. On se laisse pourtant porter par cette belle histoire. Pourquoi l'immigration devrait-elle être une expérience douloureuse ? Sophie a quitté Dakar alors que Thierno y pose le pied pour la première fois. II porte un regard émerveillé et curieux, découvre la terre de ses ancêtres, sa famille. Les cultures se bousculent. Thierno a quitté sa ville alors qu'Abdoulaye y est venu pour des raisons économiques. New York, capitale du rêve américain, ville d'immigration par excellence, condense à elle seule les deux autres pays avec son Little Italy et son Little Senegal. Sa première expérience est plutôt négative mais grâce à ses connaissances, il parvient à s'en sortir.

Ces héros issus de la classe moyenne se croisent sans jamais se rencontrer. Auréolés de bienveillance, ils cheminent vers la vie qu'ils ont choisi sans se retourner. Avec cette fiction la réalisatrice signe un tendre éloge à la liberté. A. V. 


\section{CINÉMA}

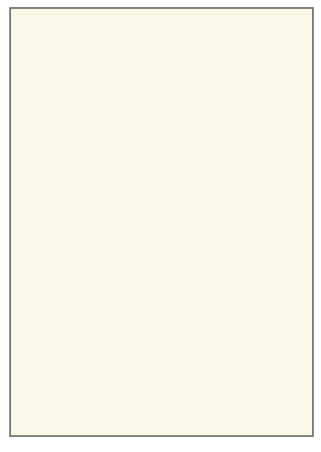

\section{Michaël Prazan}

Ellis Island, histoire d'un rêve américain

Documentaire français

Face à la statue de la liberté se dressent fièrement les imposants murs du centre fédéral d'immigration d'Ellis Island, aujourd'hui réhabilité en musée de l'immigration. Première étape d'une partie des nouveaux arrivants sur le sol américain, le centre a vu défiler de 1892 à 1954, date de sa fermeture, pas moins de douze millions d'immigrants'. Ils venaient d'Allemagne, d'Italie, de Russie... Anonymes et célébrités sont les témoins posthumes du dernier documentaire du réalisateur et écrivain Michaël Prazan diffusé sur Arte. Se croisent les destins d'un jeune immigrant irlandais William O'dwyer, futur maire de New York, ou celui d'un garçon venu de Sicile, Salvatore Luciana, surnommé plus tard Lucky Luciano, qui deviendra l'un des ténors de la mafia italienne. Ou encore celui de la polonaise Barbara Apolonia Chalupiec, la célèbre actrice plus connue sous le pseudonyme de Pola Negri, égérie de Lubitsh et première star européenne a avoir fait carrière à Hollywood. On y rencontre aussi un autre migrant polonais, Samuel Goldwyn, non moins célèbre producteur américain. À travers leurs histoires émouvantes se bâtit en trois parties chronologiques un pan de la Grande Histoire du multiculturalisme américain. Quelle a été l'importance de ce centre dans cette construction? Cet entre-deux-mondes, lieu de transit, "usine à fabriquer des citoyens américains" selon Pérec continue de fasciner. Depuis les premières images du centre par Edison en 1903, les cinéastes ont largement abordé ce sujet. On se souvient des Récits d'Ellis Island de Georges Perec et Robert Bober en 1979 et d'Ellis Island de Meredith Monk. Island of Hope - Island of Tears de Charles Guggenheim 1989. Il est aussi le théâtre de nombreuses fictions comme The Migrant de Charlie Chaplin, The Yellow Ticket de Raoul Walsh, America, America d'Elia Kazan, Le Parrain II de Coppola, Golden door de Emanuele Crialese et, très récemment, The Imigrant de James Gray. Les photos des migrants du centre de Lewis Hine et d'Augustus Frederick Sherman ont contribué à nourrir cet imaginaire collectif. II cristallise à lui seul toute l'histoire de l'immigration américaine. Mais, par sa chronologie (18921954), par ses origines essentiellement européennes, ce lieu ne représente pourtant qu'une petite partie du flux migratoire.

Michaël Prazan revient sur les événements qui jalonnent l'histoire du centre : l'évolution du contrôle des flux migratoires, les directions successives. Le destin romanesque de ces célébrités qui en ont franchi le seuil sont illustrées par de riches images d'archives et des reconstitutions malheureusement assez inutiles. Le tout entrecoupés d'interviews de spécialistes et accompagnée d'une musique racoleuse. Il réalise ainsi un film historique quelque peu académique. A. V.

1. "L'île de M. Ellis. Du dépôt de munitions au lieu de mémoire", in Hommes \& Migrations, $n^{\circ}{ }^{\circ} 1247,2004$. 


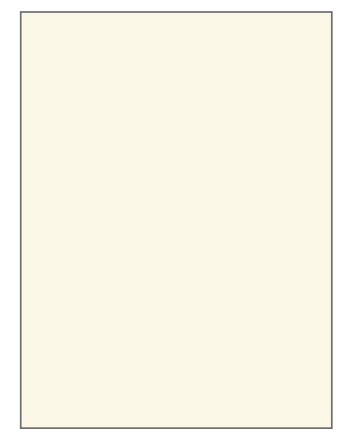

Mama Keïta

L'Absence

Drame français, guinéen, sénégalais

Présenté pour la première fois au public en 2009, le dernier film du réalisateur franco-guinéen Mama Keïta tourné avec un petit budget a mis cinq ans avant de pouvoir sortir enfin, le 22 janvier dernier, dans une seule salle parisienne. L'Absence a pourtant été sélectionné au festival de Rotterdam et a reçu le prix du meilleur scénario au Fespaco.

Ama Diop, un immigré sénégalais est de retour à Dakar. II est parti en France pour étudier et est devenu un éminent ingénieur. Débordé par sa carrière, il a délaissé sa famille et n'est pas rentré depuis quinze ans. Pour le faire revenir, sa sœur muette use d'un stratagème. Elle prétexte que leur grand-mère qui les a élevés alors qu'ils étaient devenus orphelins est souffrante. Ama se laisse berner et retrouve sa grand-mère et sa sœur toutes deux en bonne santé. Cette ruse est justifiée par une profonde détresse. Contrainte de se prostituer, la jeune fille tente un dernier appel au secours. Confronté à une bien plus sombre réalité que celle qu'il avait quitté, Ama, désemparé, se retrouve piégé dans l'étau de la corruption. II découvre alors avec son regard de jeune intellectuel une réalité amère qui le mènera jusqu'à une course poursuite haletante lancée par un proxénète d'une violence insoupçonnée. Mama Keita offre un thriller déchirant sur fond de drame familial et social. La France est la première destination pour les étudiants sénégalais. Mama Keita est venu étudier le droit à Paris avant de devenir scénariste puis, en 1981, réalisateur. Les jeunes sénégalais portent, faute de formation dans leur pays, et en raison des grèves fréquentes qui paralysent le système éducatif pendant plusieurs mois et de l'influence de l'entourage'. La France est préférée aux pays anglo-saxons pour des raisons historiques, la gratuité de son enseignement et sa langue. Des bourses subventionnées par l'État sont attribuées pour former ces jeunes à l'étranger. Mais quand ces étudiants décident de s'installer en France, le gouvernement qui a investi pour eux essuie une réelle pertefinancière ${ }^{2}$.Amaestl'und'entreeux. Une fois diplômé en France, il y est resté, car il sait qu'il ne trouvera pas de travail mieux rémunéré dans son pays d'origine. Il peut continuer ainsi à envoyer de l'argent à sa famille.Transférer del'argent déculpabilise Ama pour son absence. Son pays d'origine lui est devenu étranger.ll méprise ceuxquil'ont naguère aidé. II se moque de son ancien professeur qui l'incite à revenir s'installer à Dakar. II n'y voit que misère sociale et corruption. Il a choisi sa carrière professionnelle au détriment de sa vie familiale. II a abandonné son rôle de grand frère et ne soupçonne que trop tardivement la détresse de sa petite sœur. Dans ce polar sombre Mama Keita interroge la schizophrénie du déracinement et la déliquescence des liens familiaux. A. V.

1. Mamadou Saliou Mbengue, "Le non-retour des étudiants sénégalais formés en France : la pression familiale mise au pilori", in Seneweb.com, 2012. 2. Magatte Fall, "Migration des étudiants sénégalais", in Hommes \& migrations, $n^{\circ} 1286-1287,2010, p p$. 222-223. 